Educación Física y Ciencia, vol. 19, n² 2, e038, diciembre 2017. ISSN 2314-2561

Universidad Nacional de La Plata.

Facultad de Humanidades y Ciencias de la Educación.

Departamento de Educación Física

\title{
Perfil dermatoglífico y condición física de jugadores adolescentes de futbol
}

\author{
Dermatoglyphic profile and physical condition of adolescent soccer \\ players
}

\author{
Aldo Neyl Rodríguez Arrieta *; Oscar Alfredo Montenegro Arjona **; \\ Jorge Luis Petro Soto*
}

* Grupo de Investigación GICAFS, Universidad de Córdoba, Montería, Colombia; ** Grupo de investigación ALTIUS, Universidad Surcolombiana, Neiva, Colombia | anrodriguez@correo.unicordoba.edu.co; alfredo.montenegro@usco.edu.co; jorgelpetro@unicordoba.edu.co

\section{PALABRAS CLAVE}

Dermatoglifia

Fútbol

Condición física

\section{KEYWORDS}

Dermatoglyphics

Soccer

Physical condition

\section{RESUMEN}

El objetivo del estudio fue establecer la relación entre el perfil dermatoglífico y la condición física de los jugadores de fútbol de la selección sub-16 del Departamento de Córdoba, Colombia. Se evaluaron

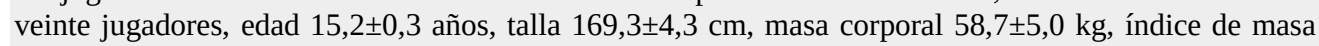
corporal $20,5 \pm 2,1 \mathrm{~kg} / \mathrm{m}^{2}$ y porcentaje de masa adiposa $10,2 \pm 2,7 \%$. Los niveles de fuerza explosiva de los miembros inferiores, de la potencia aeróbica, de la velocidad, agilidad y de la fuerza máxima de los miembros superiores, mostraron un buen desarrollo con relación a estudios nacionales e internacionales con poblaciones de la misma edad. El perfil dermatoglífico encontrado fue Arco 0,45 \pm 0,8; Presilla 6,5 \pm 2,7; Verticilo 3,0 \pm 3,0; SQTL 131,6 \pm 39.1 e Índice delta (D10) $12,6 \pm 3,5$. Se encontró una correlación muy significativa $(\mathrm{p}=<0,00)$ entre el registro de los arcos y el salto largo sin impulso (SLSI). También se encontró correlación significativa $(\mathrm{p}=<0,05)$ entre la sumatoria de la cantidad total de líneas de las dos manos (SQTL) y el SLSI y entre D10 y el SLSI; pero, no se encontró correlación significativa entre las demás características dermatoglíficas y los indicadores de las capacidades físicas.

\section{ABSTRACT}

The goal of the study was to establish the relationship between the dermatoglyph profile and the physical condition of the soccer players of the U16 team Cordoba State, Colombia. Twenty players were evaluated, age 15,2 $\pm 0,3$ years old, height $169,3 \pm 4,3 \mathrm{~cm}$, body mass $58,7 \pm 5,0 \mathrm{~kg}$, body mass index 20,5 $\pm 2,1 \mathrm{~kg} / \mathrm{m}^{2}$ and percentage of adipose mass $10,2 \pm 2,7 \%$. The levels of explosive strength of the lower limbs, aerobic power, speed, agility and maximum strength of the upper limbs, showed a good development in relation to national and international studies with populations of the same age. The dermatoglyphic profile found was: Arch 0,45 \pm 0,8; Loop 6,5 $\pm 2,7$; Whorl 3,0 $\pm 3,0$; SQTL 131,6 $\pm 39,1$ and Delta index (D10) $12,6 \pm 3,5$. A very significant correlation $(p=<0,00)$ was found between arches and Standing Long Jump (SLJ). There was also a significant correlation $(p=<0,05)$ between the sum of the quantity of total lines on both hands (SQTL) and SLJ, and between D10 and SLJ; but, no significant correlation was found between the other dermatoglyphic characteristics and the physical ability indicators. 


\section{Introducción}

De acuerdo a la revisión de antecedentes, en Colombia hay muy pocas investigaciones que informen del potencial genético por medio del estudio de la dermatoglifía deportiva. Por ejemplo, Avella \& Medellín (2013) estudiaron el perfil dermatoglífico y somatotípico de atletas de la selección Colombia de atletismo (velocidad) participante en los Juegos Panamericanos de Guadalajara 2011; Por su parte, Medellín (2014) estudió la caracterización dermatoglífica de las ciclistas Colombianas de pista de altos logros en pruebas de semifondo; Del mismo modo, Rodriguez (2014) estudió la correlación de las características genotípicos y fenotípicos de ciclistas Bogotanos en la modalidad e BMX y Hernández \& Naranjo (2016) determinaron el Perfil fenotípico y genotípico en jugadoras de fútbol; sin embargo, no se encontraron estudios que informen acerca del perfil genético por medio de las características dermatoglíficas en futbolistas nacionales de la categoría 15 años, género masculino.

Es necesario conocer muchos aspectos del fútbol, de acuerdo a como lo mencionan Castanhede, Fernandes \& Dantas (2003) y el presente estudio considera la necesidad de conocer características de la dermatoglífia deportiva del fútbol en las categorías sub 16 años; lo cual está en correspondencia con las recomendaciones del estudio realizado Dantas \& Fernandes (2002). Debido a esto, es muy relevante identificar las características cualitativas y cuantitativas de la dermatoglífia en jugadores de fútbol colombianos, para poder relacionarlas con el potencial de desarrollo de la composición corporal y de las capacidades físicas. También, para poder comparar las características dermatoglificas del grupo investigado con las características de jugadores de fútbol de alto nivel, que participan en campeonatos profesionales en Brasil (Castanhede, Fernandes \& Dantas (2003); De Almeida, Silva \& Fernandes (2005).

El estudio de las características dermatoglíficas en deportistas ha sido abordado desde hace ya un par de décadas atrás y esos estudios han sido usados para seleccionar deportistas con rendimientos sobresalientes en diferentes deportes (Zary, Reis, Rouboa, Silva, Fernandes \& Filho, 2010; Hernández, Hernández, \& Fernandes, 2013; Fernandes \& Cunha, 2004; Del Vecchio \& Goncalves, 2011; Díaz \& Espinoza, 2008). A partir de los mencionados estudios se ha creído que la impresión de la huella digital de las falanges distales de las manos sirve para evaluar el potencial físico de los deportistas, como un instrumento valioso en la orientación del rendimiento (De Almeida, Silva \& Fernandes, 2005). Por lo tanto, el método de estudio de la dermatoglífia deportiva puede ser usado como un criterio de selección y orientación deportiva, según lo menciona Abramova, Nikitina, Shafranova, Kotchetkova \& Secamova, (1996).

De acuerdo a lo anteriormente mencionado, el objetivo principal de este estudio es establecer la relación entre el perfil dermatoglífico y la condición física de los jugadores de fútbol de la Selección Córdoba y, de igual manera, se pretende identificar aspectos del nivel de desarrollo de las capacidades condicionales de la fuerza, la velocidad, la agilidad y la resistencia, en la misma población.

\section{Metodología}

La presente investigación se enmarcó bajo un enfoque cuantitativo y de un alcance correlacional (Hernández, Fernández, y Baptista, 2014), donde participaron 20 jugadores que conformaban la selección masculina de fútbol del Departamento de Córdoba - Colombia, donde participaron 20 jugadores que conformaban la selección masculina de fútbol del Departamento de Córdoba - Colombia, con una edad de 15,2 \pm 0,3 años, talla de 169,3 \pm

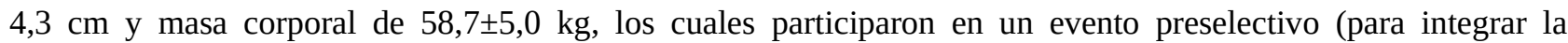
mencionada selección) denominado "Torneo de Escuelas Deportivas", organizado por la Liga de futbol de Córdoba. Después de explicar detalladamente el objetivo de la investigación, el procedimiento metodológico y los riesgos de la misma, los representantes de los adolescentes evaluados firmaron la carta de consentimiento para su participación voluntaria en el estudio. Se tuvo en cuenta la resolución 8430 del Ministerio de Salud (1993), la cual considera la presente investigación como de riesgo mínimo por tratarse de un estudio no invasivo.

Para caracterizar el grupo evaluado se tomaron algunas medidas antropométricas básicas, como son: talla y peso, bajo las normas establecidas por la Sociedad Internacional para el Avance de la Cineantropometría (ISAK), que 
están descritas en Marfell-Jones, Olds, Stewart \& Carter (2006). El índice de masa corporal (IMC) se evaluó teniendo en cuenta las tablas de referencia de la Organización mundial de la salud (OMS), que fueron adoptadas en Colombia por el Ministerio de Protección Social (2010). Para evaluar la talla se utilizó un Estadiometro Seca 206, para evaluar el peso se utilizó una Báscula digital Tanita UM-076.

De igual manera, se estimó el porcentaje de grasa corporal (masa adiposa) de los jugadores tomando como referencia la propuesta de Slaughter et al. (1988), los cuales proponen estimar el porcentaje de masa adiposa con poblaciones infanto-juvenil considerando solo pliegues tríceps y pantorrilla. La toma de los pliegues cutáneos mencionados se realizó con un calibrador marca Harpenden, que tiene una precisión en la medida de 0,2mm.

Para conocer el perfil dermatoglífico se aplicó el protocolo de dermatoglífia propuesto por Cummins \& Midlo (1961), donde se tomaron las huellas de los diez dedos de las manos de cada jugador, obteniendo el dactilograma o dibujo dactilar. Para esto se utilizó una placa de impresiones dactilares en acero inoxidable marca Sirchie FPT260, tinta para impresiones dactilares Sirchie 230T y tarjetas para registro de impresiones dactilares Sirchie FC334.

La identificación del grupo de clasificación al cual pertenece el dactilograma se realizó aplicando patrones de reconocimiento previamente establecidos, de acuerdo a la configuración de los surcos epidérmicos en la región central o nuclear del dactilograma. Para esto se empleó una lupa profesional para impresiones dactilares marca Sirchie JC101M. Los patrones de reconocimiento dermatoglifo principalmente son tres: (A) arco, (L) presilla (lazo) y (W) verticilo (espiral), que constituyen la característica cualitativa de la dermatoglifia deportiva de acuerdo a como lo menciona Del Vecchio \& Goncalves (2011) (Figura 1).

Figura 1. Patrones de reconocimiento más comunes.

\begin{tabular}{|l|l|l|}
\hline $\operatorname{Arco}(\boldsymbol{A})$ & Presilla $(\mathrm{L})$ & $\operatorname{Verticilo(W)}$ \\
\hline & & \\
& & \\
\hline & & \\
\hline
\end{tabular}

Enseguida, se procede a calcular el índice delta (D10) teniendo en cuenta los dactilogramas de los 10 dedos de las manos, donde por cada presilla (L) se cuenta un punto y por cada verticilo (W) se cuentan dos puntos (De Carvalho, Da Silva \& Fernandes, 2005). En el índice delta el valor mínimo es cero "0” y el valor máximo es veinte "20", donde el valor cero es justificado con la presencia de arcos (A) y la ausencia de deltas.

Posteriormente, se halla la sumatoria de la cantidad total de líneas papilares (SQTL) de todos los dedos en las dos manos. Para esto, se debe determinar el número de líneas existentes únicamente en el núcleo de las presillas (L) y en el núcleo de los verticilos (W) por la línea de Galton, la cual es una línea imaginaria que tiene como punto de partida el delta y el de llegada el centro del núcleo, que se sitúa en el ápice (extremo superior o punta) de una presilla, Figura 2. De acuerdo con diversos autores, la SQTL constituye la característica cuantitativa de la Dermatoglifía deportiva (Da Cunha \& Filho, 2004; Del Veccino \& Goncalves, 2011; Diaz \& Espinosa, 2008; Cunha-Montenegro et al., 2013). 
Figura 2. Trazo de la línea de Galton en una presilla (L).

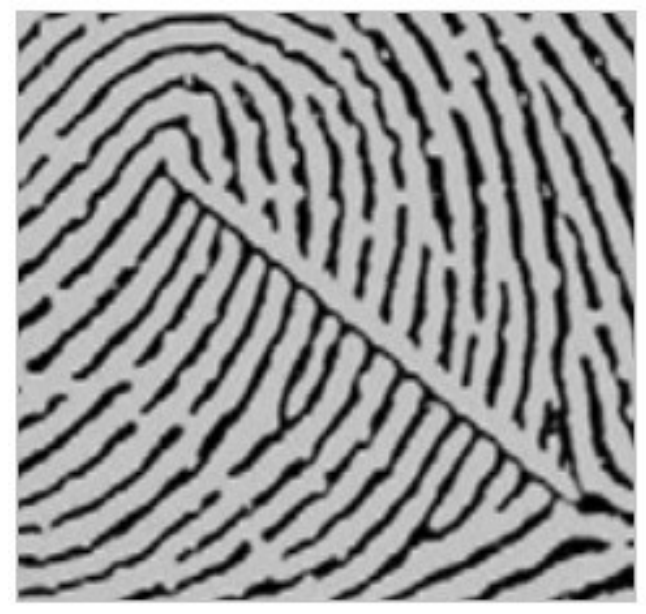

En el conteo de las crestas papilares por la línea de Galton, se adoptó el método de Vucetich; es decir, se establece la cantidad de líneas existentes en el dactilograma que están atravesadas por la línea de Galton, sin tener en cuenta el punto de partida (punto déltico) y el punto de llegada (punto nuclear), de acuerdo a como lo referencian Cummins \& Midlo (1961) y Zary et al., (2010).

Las características cualitativas y cuantitativas de los patrones dermatoglíficos son marcadores objetivos para la orientación y selección en el deporte. Abramova, et al. (1996) realizaron un análisis y clasificación de los índices somático-funcionales entre atletas de alto rendimiento en Rusia, llegando a estructurar cinco principales grupos, tabla 1.

Tabla 1. Clasificación del conjunto de los índices dermatoglíficos e índices somatico-funcionales en atletas rusos de alto rendimiento $(\mathrm{n}=600)$.

\begin{tabular}{|c|c|c|l|l|}
\hline \multirow{2}{*}{ Clase } & \multicolumn{2}{|c|}{ Impresiones Digitales } & \multicolumn{2}{c|}{ Características Funcionales } \\
\cline { 2 - 5 } & D10 & SQTL & \multicolumn{1}{c|}{ Nivel mínimo } & \multicolumn{1}{|c|}{ Nivel máximo } \\
\hline I & 5,5 & 28 & $\begin{array}{l}\text { Estatura, Fuerza Absoluta, } \\
\text { Resistencia, Coordinación. }\end{array}$ & Fuerza Relativa \\
\hline II & 6,0 & 48 & Coordinación & Fuerza \\
\hline III & 11,6 & 126 & Fuerza absoluta y fuerza relativa & Estatura \\
\hline IV & 13,1 & 134 & Estatura, Fuerza Absoluta & Coordinación y Resistencia \\
\hline V & 17,5 & 163 & Fuerza Relativa & Coordinación. \\
\hline
\end{tabular}

Para la interpretación de los diseños dermatoglíficos Hernández, Hernández \& Fernandes (2013), describen la siguiente clasificación.

a. El nivel bajo del índice delta (D10), el aumento de los dibujos del Arco (A) y Presilha (L), la disminución de dibujos de Verticilo (W) y la disminución de la suma total de cantidad de líneas en todos los dedos (SQTL), todos son propios de las modalidades deportivas, con la potencia alta y el tiempo corto de duración;

b. El nivel alto de D10, la falta del arco (A), el aumento de la proporción del verticilo (W) el aumento SQTL, caracteriza a las modalidades deportivas de resistencia de velocidad. Las modalidades de juegos deportivos demuestran esta tendencia; Las modalidades de deportes de velocidad y de fuerza, ellos se insertan en el campo de valores bajos de D10 y de SQTL.

c. En las modalidades, con la propiocepción compleja, se hallan en el campo de altos valores de D10. 
d. En los grupos de deportes de resistencia, el D10 ocupa la posición intermedia.

Para la evaluación de la condición física se aplicaron diferentes test de acuerdo a la capacidad a evaluar, así: para la evaluación de la fuerza explosiva de miembros inferiores se realizó el test de salto largo sin impulso (SLSI) de acuerdo con el protocolo propuesto por Amuzaini \& Fleck (2008). Los jugadores calzaron guayos para realizar el test, se marcó una línea de salida, la superficie de caída fue sobre césped donde se extendió longitudinalmente en la dirección del salto una cinta métrica de acero marca Komelon 200' Steel Reel, calibrada a $50 \mathrm{~N}$ y $20^{\circ} \mathrm{C}$. La lectura se realizó de manera directa con una precisión en la lectura hasta el centímetro más cercano a la línea de salida, haciendo una medición perpendicular desde el talón de la huella de caída hasta la cinta métrica extendida en el suelo. El mejor de tres saltos fue usado para el análisis. La confiabilidad de esta técnica de medición del SLSI es muy alta (ICC=0.97), como lo mencionan Amuzaini \& Fleck (2008).

La evaluación de la velocidad se realizó con una carrera sobre una distancia de 50 metros, tomando tiempos parciales cada 10 metros de acuerdo a como lo proponen Montenegro \& Ramos (2015), Vescovi (2012) y Jastrzebski et al. (2013). Los tiempos de carrera se tomaron manualmente por parte de un experto con un cronómetro Casio HS-70W, donde el tiempo para completar cada intento fue registrado en segundos y centésimas de segundo. Los jugadores realizaron dos intentos con un descanso de 5 minutos entre cada uno, para asegurar una recuperación completa entre los intentos. El mejor de los dos resultados fue usado para el análisis.

La evaluación de la fuerza máxima de las extremidades superiores se determinó mediante el test de dinamometría manual descrito por Das \& Dutta (2015). Se utilizó un dinamómetro Jamar SP-5030J1, donde se incluyó un ajuste previo del agarre del dinamómetro en función del tamaño de la mano, para así poder obtener el mejor resultado en la prueba. El agarre óptimo en función del tamaño de la mano se calcula mediante la ecuación propuesta por Ruiz et al. (2006), la cual es: $\mathrm{Y}=\mathrm{X} / 7,2+3,1$; donde $\mathrm{X}$ es el tamaño de la mano, medido entre el primer y quinto dedo (teniendo la mano totalmente extendida y los dedos separados lo más posible) e $\mathrm{Y}$ es el agarre a utilizar. El evaluado aprieta de forma gradual y continua durante al menos $2 \mathrm{~s}$, realizando la prueba una vez con la mano derecha y una vez con la mano izquierda. El máximo valor en kilogramos para cada mano fue tenido en cuenta, registrando el valor con una precisión de 0,1 kg. El promedio del valor alcanzado en las dos evaluaciones de fuerza máxima prensil fue usado para el análisis. La confiabilidad de esta técnica de medición de la fuerza máxima de las extremidades superiores es aceptable, como lo mencionan Ortega et al. (2008).

Para determinar la capacidad de acelerar, decelerar, girar en diferentes direcciones y ángulos se eligió el test de agilidad de Illinois (IAT), el cual se administró utilizando una versión estandarizada reportada por Roozen (2004), Raya et al. (2013) y Alanazi (2015). La longitud del recorrido fue marcada por cuatro conos ubicados en las esquinas de un rectángulo de $10 \mathrm{~m}$ de largo y $5 \mathrm{~m}$ de ancho, otros cuatro conos fueron ubicados en el centro, separados 3,3 m entre sí y colocados a 2,5 m de los conos ubicados a lo ancho. El jugador comenzó la prueba en posición de cúbito abdominal detrás de la línea de salida con los brazos a su lado y la cabeza girada al lado o hacia adelante. A la señal de partida, el jugador corre y acelera rápidamente haciendo un recorrido de ida hacia la primera marca de cinta en el suelo, debe tocar o cruzar la marca de la cinta con su pie. Luego, el jugador gira y corre de vuelta hacia el primer cono del centro; allí, gira para hacer un recorrido de ida en zig-zag por entre los cuatro conos ubicados en el centro. Después, el jugador gira y de nuevo hace un recorrido de vuelta en zig-zag por entre los cuatro conos ubicados en el centro. Enseguida, el jugador gira y corre de ida lo más rápido posible hacia la segunda marca de cinta en el suelo. Por último, el jugador gira y hace un recorrido de vuelta corriendo lo más rápido posible para pasar a través de la línea final. El tiempo para completar cada intento es registrado en segundos y centésimas de segundo. Si un jugador no realiza el recorrido según las instrucciones, no toca con su pie las marcas hechas con cinta en el piso, no completa el recorrido o mueve alguno de los conos, debe repetir la prueba.

Para la evaluación de la potencia aeróbica se aplicó el test propuesto por Leger, Mercier, Gadoury \& Lambert (1988), el cual puede estimar de manera indirecta el consumo máximo de oxígeno. El test consiste en una prueba progresiva, que se realiza mediante carreras de ida y vuelta sobre una distancia de $20 \mathrm{~m}$, manteniendo el ritmo con señales auditivas emitidas por un CD pregrabado. La velocidad inicial es de $8.5 \mathrm{~km} \mathrm{~h}^{-1}$, la cual se incrementa 0.5 
$\mathrm{km} \mathrm{h}^{-1} \mathrm{~min}^{-1}$, donde un minuto equivale a una etapa. El test se termina cuando el participante no llega a las líneas finales simultáneamente con la señal auditiva en dos ocasiones. Los participantes fueron motivados para seguir corriendo el mayor tiempo posible durante toda la prueba. Se realizó un único intento para ser tenido en cuenta en la evaluación, donde el final de la última etapa en la que el participante se retiró se tuvo en cuenta para su evaluación. Para calcular el consumo máximo de Oxígeno $\left(\mathrm{VO}_{2 \text { máx }}\right)$, se empleó la siguiente formula:

$$
\mathrm{VO}_{2 \text { máx }}\left(\mathrm{mL} \cdot \mathrm{kg}^{-1} \mathrm{~min}^{-1}\right)=31,025+(3,238 \text { x VE) }-(3,248 \text { x E) }+(0,1536 \text { x VE x E) }
$$

Donde: VE: Velocidad de la última etapa que pudo culminar el evaluado; E: Edad en años.

Con la información recolectada con las distintas técnicas de medición, se procedió a conformar una matriz de datos con el programa Microsoft Excel 2013M , posteriormente, se realizó el análisis estadístico con el programa estadístico SPSS versión 20, con el cual se llevaron a cabo pruebas descriptivas de tendencia centralizada (e.g. media) y de variabilidad (desviación estándar y coeficiente de variabilidad). De igual forma, se aplicaron pruebas estadísticas para establecer la correlación entre las variables con el test de correlación Pearson o rho de Spearman, en dependencia a la normalidad de los datos. La normalidad de los datos se obtuvo con la prueba de Shapiro-Wilk.

\section{Resultados y discusión}

La selección de la muestra se realizó de forma no probabilística, la cual estuvo conformada por jóvenes de la selección de fútbol sub 16 del Departamento de Córdoba, Colombia $(\mathrm{n}=20)$. La edad de los jugadores fue $15,2 \pm 0,3$ años, con una talla de $169,3 \pm 4,3 \mathrm{~cm}$, una masa corporal de 58,7 $\pm 5,0 \mathrm{~kg}$, un índice de masa corporal de $20,5 \pm 2,1 \mathrm{~kg} / \mathrm{m}^{2}$ y un porcentaje de masa adiposa de 10,2 $\pm 2,7 \%$. Con relación a los indicadores antropométricos, aquellos que relacionan la masa corporal y la talla, como el IMC, muestran en término general, que los jóvenes evaluados se encuentran dentro de los valores normales para la edad, es decir: peso corporal adecuado para la edad, una talla adecuada para la edad y un IMC adecuado para la edad, en vista que los valores de los indicadores relacionados se encontraron dentro del punto de corte ( $\geq-1$ a $\leq 1$ desviación estándar) establecido por la OMS (De Onís et al., 2007).

En el estudio de la composición corporal, en particular, respecto al porcentaje adiposo o de masa grasa, en este estudio se encontraron valores que están dentro de los valores de la población de la misma edad y sexo. Tabla 2.

Tabla 2. Indicadores antropométricos.

\begin{tabular}{|c|c|c|c|c|}
\hline \multirow{2}{*}{ Variable } & \multirow{2}{*}{ Media } & \multirow{2}{*}{$\begin{array}{c}\text { Desviación } \\
\text { Estándar }\end{array}$} & \multicolumn{2}{|c|}{ Int. Conf. media 95\% } \\
\cline { 4 - 5 } & & inferior & superior \\
\hline Edad (años) & 15,2 & 0,3 & 15 & 15,3 \\
\hline Talla (cm) & 169,3 & 4,3 & 167,2 & 171,3 \\
\hline Masa Corporal (kg) & 58,7 & 4,3 & 56,4 & 61 \\
\hline Índice IP & 43,6 & 5 & 42,9 & 44,4 \\
\hline IMC $\left(\mathrm{kg} / \mathrm{m}^{2}\right)$ & 20,5 & 1,6 & 19,6 & 21,5 \\
\hline M. Adiposa & 10,2 & 2,1 & 8,9 & 11,4 \\
\hline
\end{tabular}

El estudio de la Dermatoglifia, Tabla3, desde la identificación de variables cualitativas, en el análisis estadístico y diseño de los 10 dedos, se evidencia una tendencia de presillas en todos los evaluados, seguido de verticilos y una pequeña minoría de arcos. Esto representa, según los estudios de Hernández, Hernández \& Fernandes (2013), que el grupo evaluado presenta un alto desarrollo de la velocidad, con relación a los valores de presillas $(6,5 \pm 2,7)$, de igual forma la presencia de Verticilos $(3,0 \pm 3,0)$, los relaciona a una alta coordinación motora y resistencia y una 
baja presencia de Arcos $(0,4 \pm 0,8)$, representa niveles disminuidos de la “fuerza pura” (según teoriza este método).

El conteo de D10 $(12,6 \pm 3,5)$ se asocia a los altos niveles de Resistencia y Agilidad y la suma total de líneas dérmicas en los 10 dedos (SQTL) 131,6 ×39,1 a las capacidades condicionantes de Coordinación y Resistencia.

Tabla 3. Indicadores de la dermatoglifia relacionados con la condición física.

\begin{tabular}{|c|c|c|c|c|}
\hline \multirow{2}{*}{ Variable } & \multirow{2}{*}{ Media } & \multirow{2}{*}{$\begin{array}{c}\text { Desviación } \\
\text { Estándar }\end{array}$} & \multicolumn{2}{|c|}{ IC media 95\% } \\
\cline { 4 - 5 } & & & inferior & Superior \\
\hline Arcos & 0,4 & 0,8 & 0,3 & 0,8 \\
\hline Presillas & 6,5 & 2,7 & 5,2 & 7,7 \\
\hline Verticilos & 3,0 & 3,0 & 1,6 & 4,4 \\
\hline SQTL & 131,6 & 39,1 & 113,3 & 149,9 \\
\hline D10 & 12,6 & 3,5 & 10,9 & 14,2 \\
\hline
\end{tabular}

Este perfil de rendimiento físico y dermatológico está muy relacionado con las demandas fisiológicas del fútbol, el cual se caracteriza por acciones motrices de carácter intermitente, donde se conjugan distintas capacidades motrices como la velocidad y la coordinación. Este deporte, en el alto rendimiento, se caracteriza por una baja presencia de arco (Castanhede, Fernandes, \& Dantas, (2003), de igual manera a como se halló en este estudio, donde se encontró un porcentaje del $4.5 \%$ del total de todos los dactilogramas estudiados. Recordemos que el patrón dermatoglífico arco (A) no está presente en el alto rendimiento o se encuentra muy poco y su presencia se relaciona con el bajo rendimiento deportivo, según lo reporta Abramova, et al (1996), Castanhede, Fernandes, \& Dantas, (2003) y Hernández, Hernández, \& Fernandes, (2013).

En este sentido, de acuerdo con los resultados de los indicadores dermatoglíficos, se pudo establecer que el grupo de adolescentes futbolistas presenta una alta distribución de presillas, lo cual según estudios como los de Silva, Vianna, Gomes \& Dantas (2008) está asociada con la velocidad. Además, una notable presencia de D10 y de SQTL representa maximizados la capacidad de coordinación motora y la capacidad de resistencia, como sucede en el grupo de deportes de resistencia a la velocidad, al cual pertenece el fútbol.

De otro lado, Castanhede, Fernandes \& Dantas (2003), evaluaron a 48 jugadores de la liga de fútbol profesional de Brasil que compitieron en ese país durante la temporada 2001/2002, encontrando que la presencia del patrón dermatoglífico arco es de $0,5 \pm 1.3$, el patrón presilla es $6,9 \pm 2,7$, el patrón verticilo es 2,56 $\pm 2,7$ y el índice delta D10 es de 12,0 $\pm 3,2$. Estos valores medianos de las características dermatoglíficas mencionadas, se asemejan bastante a los valores encontrados en el presente estudio, que respectivamente son: 0,4 \pm 0,8 para el arco, 6,5 +/2,7 para las presillas, 3,0 \pm 3,0 para los verticilos y 12,6 \pm 3,5 para el índice delta D10.

De acuerdo con la clasificación del conjunto de los índices dermatoglíficos y de los índices somático y funcionales para deportistas, propuestos por Abramova, et al (1996), la clase mostrada por los deportistas es la III y la IV. De igual manera se destaca el desarrollo de la resistencia y la coordinación y se tendría que trabajar más la fuerza (relativa y absoluta). No obstante, la fuerza explosiva evaluada con el SLSI se expresó en un buen nivel de desarrollo.

En cuanto los indicadores de condición física, se destaca un buen desarrollo de la resistencia, la fuerza explosiva y la velocidad. Si tomamos como referencia el estudio realizado por Salleg \& Petro (2012), en población de niños y adolescentes escolarizados de la ciudad de Montería, la velocidad en 50 metros se encuentra en el percentil 95, el SLSI en el percentil 99 y la prueba de potencia aeróbica, respecto al $\mathrm{VO}_{2 \text { máx }}$ se encuentra en el percentil 99. Tomando en cuenta lo anterior, y con base la categoría de los registros percentilares de las pruebas físicas de Lefevre, Beunen, Steens \& Claessens (1990), la capacidad de velocidad de los adolescentes evaluados se podría considerar como "muy buena" mientras que sus niveles de fuerza explosiva de miembros inferiores y de potencia 
aeróbica se consideran como “extremadamente buenos”.

De igual manera, con respecto al estudio realizado por Ortega, et al. (2011), donde evaluaron los niveles de rendimiento físico en adolescentes en más de diez ciudades europeas, el SLSI se encuentra por encima del percentil 80, la prueba de dinamometría manual está en un percentil 70 y la prueba de potencia aeróbica se encuentra en percentil 90, lo que se podría evaluar como "bueno", "bueno sobre la media” y "muy bueno" respectivamente, de acuerdo con la propuesta de Lefevre, et al, (1990).

Desde otro punto de vista, si comparamos los resultados hallados con el estudio hecho a un grupo de jugadores élite de la misma categoría (Zalai et al., 2015), encontramos que, en la prueba de SLSI los resultados (2,2 $\pm 0,0 \mathrm{~m}$ ) están, en promedio, por encima del mencionado estudio $(2,1 \pm 0,1 \mathrm{~m})$. Situación que ocurre al contrario en la prueba de carrera de $30 \mathrm{~m}$, donde los resultados de los jugadores de élite de la misma categoría $(4,4 \pm 0,2 \mathrm{~s})$ son ligeramente más rápidos con respecto al presente estudio $(4,6 \pm 0,1 \mathrm{~s})$.

Con relación a los resultados del test IAT muestran un desarrollo de la agilidad con valoración de promedio, con respecto a la tabla de evaluación que presenta Roozen (2004). Sin embargo, los resultados del grupo evaluado $(17,0 \pm 0,5 \mathrm{~s})$ son superiores a los valores reportados por Alanazi (2015) con jugadores elite de la categoría adulta $(18,0 \pm 0,6 \mathrm{~s})$.

Con estos resultados se podría plantear que estos jugadores tienen desarrolladas capacidades de bases que, mediante un entrenamiento específico, se potenciaría el talento hacia formación especializada del fútbol, atendiendo las demandas fisiológicas de este deporte.

Los Indicadores de la condición física, evidencian una alta caracterización de las capacidades de velocidad (D.T 0,2) y agilidad (D.T 0,4) (velocidad y coordinación, principalmente); identificando la resistencia (D.T 4,4) desde una baja desviación típica, mientras se plasma una marcada dispersión a la baja en el grupo, menor al $10 \%$ (coeficiente de variación), exceptuando la fuerza prensil que fue del 11\%. Todo ello marca unos altos valores de resistencia fuerza, velocidad y agilidad, para la edad y sexo.

Tabla 4. Indicadores de la condición física.

\begin{tabular}{|c|c|c|c|c|}
\hline \multirow{2}{*}{ Variable } & \multirow{2}{*}{ Media } & \multirow{2}{*}{$\begin{array}{c}\text { Desviación } \\
\text { Estándar }\end{array}$} & \multicolumn{2}{|c|}{ IC media 95\% } \\
\cline { 4 - 5 } & & inferior & superior \\
\hline $\begin{array}{c}\mathrm{VO}_{2 \mathrm{máx} .} \\
\left(\mathrm{mL} \cdot \mathrm{kg}^{-1} \cdot \mathrm{min}^{-1}\right)\end{array}$ & 54,1 & 4,4 & 52,0 & 56,2 \\
\hline SLSI (cm) & 2,2 & 0,0 & 2,1 & 2,2 \\
\hline Dinamometría (kg) & 42,7 & 4,6 & 40,5 & 44,9 \\
\hline $50 \mathrm{~m} \mathrm{(m/s)}$ & 7,1 & 0,2 & 7,0 & 7,3 \\
\hline $30 \mathrm{~m}(\mathrm{~m} / \mathrm{s})$ & 4,6 & 0,1 & 4,6 & 4,7 \\
\hline Test Illinois (s) & 16,9 & 0,4 & 16,7 & 17,2 \\
\hline
\end{tabular}

Las correlaciones entre la frecuencia de los principales indicadores dermatoglificos y los de capacidades físicas analizadas en el presente estudio, tabla 5, se destaca que solo se encontró correlación negativa $(-, 595)$ con un p valor de 0,00 entre la frecuencia de Arcos y el SLSI; lo cual confirma que el rendimiento físico es inversamente proporcional a la presencia del patrón dermatoglifo de arco (Abramova, et al, 1996, Castanhede, Fernandes, \& Dantas, 2003). En el mismo sentido, se encontró una correlación positiva entre el registro de la SQTL y el SLSI $(\mathrm{p}=, 02)$ y entre el SLSI y el índice D10 ( $\mathrm{p}=, 03)$; ante lo cual podemos inferir que la ausencia de arco (A) y un nivel alto de D10 y SQTL son característicos de los juegos deportivos donde se manifiesta la resistencia de velocidad (Hernández, Hernández \& Fernandes, 2013). De otro lado, no se encontró correlación con las demás 
variables, lo que representa la necesidad de seguir estudiando estas variables más profundamente.

Tabla 5. Correlación entre indicadores dermatoglíficos y de capacidad física.

\begin{tabular}{|c|c|c|c|c|c|}
\hline Variable & Arcos & Presillas & Verticilos & SQTL & D10 \\
\hline $\begin{array}{c}\mathrm{VO}_{2 \mathrm{máx}} \\
\left(\mathrm{mL} \cdot \mathrm{kg}^{-1} \cdot \mathrm{min}^{-1}\right)\end{array}$ & $\mathrm{r}=-, 025$ & $\mathrm{p}=0,91$ & $\mathrm{r}=-, 138$ & $\mathrm{p}=0,56$ & $\mathrm{r}=, 132$ \\
$\mathrm{p}=0,57$ & $\begin{array}{c}\mathrm{r}=-, 025 \\
\mathrm{p}=0,91\end{array}$ & $\begin{array}{c}\mathrm{r}=, 120 \\
\mathrm{p}=0,61\end{array}$ \\
\hline $\begin{array}{c}\text { SLSI } \\
(\mathrm{cm})\end{array}$ & $\mathrm{r}=-, 595$ & $\mathrm{r}=-, 220$ & $\mathrm{r}=, 372$ & $\mathrm{r}=, 497$ & $\mathrm{r}=, 469$ \\
\hline $\begin{array}{c}\text { Dinamometría } \\
(\mathrm{kg})\end{array}$ & $\mathrm{r}=-, 00 * *$ & $\mathrm{p}=0,35$ & $\mathrm{p}=0,10$ & $\mathrm{p}=0,02 *$ & $\mathrm{p}=0,03^{*}$ \\
\hline $50 \mathrm{~m}$ & $\mathrm{p}=0,25$ & $\mathrm{r}=-, 056$ & $\mathrm{r}=, 129$ & $\mathrm{r}=, 175$ & $\mathrm{r}=, 178$ \\
$(\mathrm{~m} / \mathrm{s})$ & $\mathrm{p}=0,264$ & $\mathrm{r}=, 104$ & $\mathrm{r}=-, 170$ & $\mathrm{r}=-, 139$ & $\mathrm{r}=-, 212$ \\
\hline $30 \mathrm{~m}$ & $\mathrm{r}=, 345$ & $\mathrm{p}=0,66$ & $\mathrm{p}=0,47$ & $\mathrm{p}=0,55$ & $\mathrm{p}=0,37$ \\
$(\mathrm{~m} / \mathrm{s})$ & $\mathrm{p}=0,13$ & $\mathrm{p}=0,107$ & $\mathrm{r}=-, 197$ & $\mathrm{r}=-, 165$ & $\mathrm{r}=-, 256$ \\
\hline Test Illinois & $\mathrm{r}=-, 050$ & $\mathrm{r}=, 346$ & $\mathrm{p}=0,40$ & $\mathrm{p}=0,48$ & $\mathrm{p}=0,27$ \\
$(\mathrm{~s})$ & $\mathrm{p}=0,83$ & $\mathrm{p}=0,13$ & $\mathrm{p}=0,299$ & $\mathrm{r}=-, 095$ & $\mathrm{r}=-, 245$ \\
& & & & $\mathrm{p}=0,68$ & $\mathrm{p}=0,29$ \\
& & & & \\
\hline
\end{tabular}

* La correlación es significativa al nivel 0,05 (bilateral).

** La correlación es significativa al nivel 0,01 (bilateral).

\section{Conclusiones}

Los indicadores antropométricos de masa corporal, talla, el índice de masa corporal y el porcentaje de masa adiposa, se encuentran dentro de los valores de normalidad para la población del mismo sexo y edad, de acuerdo con las tablas de referencia de la Organización Mundial de la Salud (De Onís et al., 2007).

Los niveles de fuerza explosiva de los miembros inferiores, de la potencia aeróbica, de la velocidad y de la fuerza máxima de los miembros superiores, mostraron un buen desarrollo con relación a estudios nacionales e internacionales con poblaciones de la misma edad. Así mismo, los resultados de la prueba de agilidad se encuentran por encima de los valores reportados por Alanazi (2015), con jugadores elite de la categoría adulta.

El patrón dermatoglífico mostrado por el grupo muestra un marcado registro de los indicadores analizados (comparados con estudio de referencia en el área), especialmente de presilla, D10 y SQTL. Los resultados son coherentes con los niveles de desarrollo de los indicadores de las capacidades físicas, en especial la resistencia y la velocidad.

Se establece que el perfil dermatoglífico encontrado en la población evaluada es: Arco 0,45 \pm 0,8; Presilla 6,5 \pm 2,7; Verticilo 3,0 \pm 3,0; SQTL 131,6 \pm 39.1 y D10 12,6 \pm 3,5. El predominio de presillas nos indica una facilidad para la hipertrofia muscular y una predisposición para el desarrollo de la velocidad y fuerza explosiva (Fernandes, 2010).

La clasificación del conjunto de los índices dermatoglíficos y de los índices somático- funcionales para deportistas en el grupo evaluado es la III y la IV (Abramova et al., 1996), donde se infiere que tienen de moderada a alta predisposición para el alto rendimiento.

Se encontró una correlación positiva entre el registro de la SQTL y el SLSI y entre el SLSI y el índice D10. También se encontró una correlación negativa entre la prueba de SLSI y la frecuencia de los arcos; pero, no se encontró correlación significativa entre las demás características dermatoglíficas y los indicadores de las capacidades físicas. 


\section{Referencias}

Abramova, T., Nikitina, T., Shafranova, E., Kotchetkova, N. \& Secamova, G. (1996). “Finger Dermatoglyphs as Markers of the Funtional Features”. En: Rogozkin, V. \& R. Maughan, R. (Ed), Current Research in Sport Sciences (pp.213-216). New York, United States: Plenum Press.

Alanazi, M. (2015). Relationships between illinois agility test and reaction time in male athletes. The Swedish Journal of Scientific Research, 2(3), 28-33.

Amuzaini, K. \& Fleck, S. (2008). Modification of the standing long jump test enhances ability to predict anaerobic performance. Journal of Strength and Conditioning Research, 22(4), 1265-1272. doi: 10.1519/JSC.0b013e3181739838.

Avella, R. \& Medellín, J. (2013). Perfíl Dermatoglífico y Somatotípico de Atletas de la Selección Colombía de Atletismo (Velocidad) Participantes en los Juegos Panamericanos de Guadalajara, 2011. Revista UDCA Actualidad \& Divulgación Científica, 16(1), 17-25.

Castanhede, A., Fernandes, J. \& Dantas, P. (2003). Dermatoglyphic and Somatotype profile of male soccer athletes of high performance in Rio de Janeiro-Brazil. Journal of Fitness and Performance, 2(4), 234-239.

Cummins, H. \& Midlo, C. (1961). Palmar and Plantar Dermatoglyphics in primates. An Introduction to Dermatoglyphics. New York, United States: Dover Publications, Inc.

Cuncha Montenegro, R., Renato Paz, C., De Lucena Barbosa, E., Nóbrega Montenegro Neto, A., Salles De Oliveira, M., Soares De Araújo Filho, V., ... Fernandes Filho, J. (2013). Association between Dermatoglyphic Configuration and the ACTN3 Genotype in Juvenile Male Athletes. Croatian Journal of Education, 3(4), 11-29.

Da Cunha, R. \& Filho, J. (2004). Identification of dermatoglyphic profile of high level foreign fencers of the three armed force, participants of fencing world championship-Havana-Cuba/ 2003. Fitness \& Performance Journal, 3(5), 247-253.

Dantas, P. \& Fernandes, J. (2002). Identification of the profiles, genetic, of physical fitness and somatical type that characterize masculine athletes, of high income, participants of adult futsal, in Brazil. Fitness and Performance Journal, 1(1), 28-36.

Das, A. \& Dutta, M. (2015). Correlation between Body Mass Index and Handgrip Strength and Handgrip Endurance among Young Healthy Adults. Journal of Evidence based Medicine and Healthcare, 2(27), 3995-4001.

De Almeida, M., Silva, P. \& Fernandes, J. (2005). Relación de los índices dermatoglíficos con evaluación isocinética y la ergometría con consumo de oxígeno. Journal of Fitness and Performance, 4(2), 101-106.

De Carvalho, E., Da Silva, J. \& Fernandes, F. (2005). Dermatoglyphic, somatotypic and physiologic profiles of athletes of high performance, participants of resistance race, in Rio de Janeiro. Journal of Fitness and Performance, 4(3), 168-174.

De Onís, M., Onyango, A., Borghi, E., Siyam, A., Nishida, C. \& Siekmann, J. (2007). Development of a WHO growth reference for school-aged children and adolescents. Bulletin of the World Health Organization, 85(9), 660667. doi:10.2471/BLT.07.043497

Del Vecchio, F. \& Goncalves, A. (2011). Dermatoglifos como indicadores biológicos del rendimiento deportivo. Revista Andaluza de Medicina del Deporte, 4(1), 38-46.

Díaz, J. \& Espinoza, O. (2008). Dactiloscopy and physical fitness of members of the Center of Initiation and Expertise in Athletics of the First Region. Fitness and Performance Journal, 7(4), 209-216. 
Fernandes, J. (2010). La impresión digital (dermatóglifos) y la detección de talenyos deportivos. ISBN 978-85909946-2-6. Curso de Dermatoglifia Deportiva. Memorias. Soacha, Colombia: Universidad de Cundinamarca.

Hernández, C. \& Naranjo, R. (2016). Determinación del Perfil Fenotípico y Genotípico en Jugadoras Bogotanas del Club Gols Star. Revista Digital Actividad Física y Deporte, 2(1), 22-36.

Hernández, C., Hernández, D. \& Fernandes, J. (2013). Perfil dermatoglifico de jugadores profesionales de futbol del Club Deportivo Ñublense de la Ciudad de Chillan. Revista Motricidad Humana, 14(1), 9-15.

Hernández, R., Fernández, C., y Baptista, M. (2014). Metodología de la Investigación, Sexta Edición. México D.F., México: McGraw-Hill Interamericana.

Jastrzebski, Z., Radziminski, L., Dargiewicz, R., Jaskulska, E., Barnat, W. \& Rompa, P. (2013). Generic versus specific sprint training in young soccer players. Baltic Journal of Health and Physical Activity, 5(3), 191-198.

Lefevre, J. (1990). Norm scales and profile charts for anthropometric measurements, motor fitness, physiological measurements, and skeletal maturity. En Simons, J. et al. (Ed), Growth and fitness of Flemish Girls. Champaign, United States: Human Kinetics.

Lefevre, J., Beunen, G., Steens, G. \& Claessens, A. (1990). Motor performance during adolescence and age thirty as related to age at peak height velocity. Annals of Human Biology, 17(5), 423-435.

Leger, L., Mercier, D., Gadoury, C. \& Lambert, J. (1988). The multistage 20 metre shuttle run test for aerobic fitness. Journal of sports sciences, 6(2), 93-101.

Marfell-Jones, M., Olds, T., Stewart, A. \& Carter, L. (2006). International Standards for Anthropometric Assesment. (N.-W. U. Press, Ed.) South Africa: International Society for the Advancement of Kinanthropometry ISAK.

Medellín, J. (2014). Caracterización Dermatoglífica de las Ciclistas Colombianas de Pistas de Altos Logros en Pruebas de Semifondo. Revista UDCA Actualidad \& Divulgación Científica, 17(1), 45-52.

Ministerio de Salud (1993). Resolución Número 8430. Colombia, 4 de octubre de 1993.

Ministerio de Protección Social (2010). Resolución número 2121. Colombia, 9 de Junio de 2010.

Montenegro, O. \& Ramos, S. (2015). La velocidad en el niño. Ritmo y Diferenciación. Armenia, Colombía: Editorial Kinesis.

Ortega, F., Artero, E., Ruiz, J., España-Romero, V., Jiménez-Pavón, D., Vicente-Rodríguez, G., ... Castillo, M. (2011). Physical fitness levels among European adolescents: the HELENA study. British Journal of Sports Medicine, 45(1), 20-29. doi:10.1136/bjsm.2009.062679

Ortega, F., Artero, E., Ruiz, J., Vicente-Rodriguez, G., Bergman, P., Hagstromer, M., ...Castillo, M. (2008). Reliability of health-related physical fitness tests in European adolescents. The HELENA Study. International Journal of Obesity, 32(5), 49-57. doi:10.1038/ijo.2008.183

Raya, M., Gailey, R., Gaunaurd, I., Jayne, D., Campbell, S., Gagne, E., . . Tucker, C. (2013). Comparison of three agility tests with male servicemembers: Edgren Side Step Test, T-Test, and Illinois Agility Test. Journal of Rehabilitation Research and Development, 50(7), 951-960.

Rodríguez, A. (2014). Correlación de las características genotípicos y fenotípicos de ciclistas Bogotanos en la modalidad de BMX. (tesis de maestría). Universidad Pedagógica y Tecnológica de Colombia, Tunja, Colombia.

Roozen, M. (2004). Illinois agility test. NSCA’s Performance Training Journal, 3(5), 5-6.

Ruiz, J., España-Romero, V., Ortega, F., Sjöström, M., Castillo, M. \& Gutierrez, A. (2006). Hand Span Influences Optimal Grip Span in Male and Female Teenagers. The Journal of Hand Surgery, 31(A), 1367-1372. 
Salleg, M. \& Petro, J. (2012). Perfil de aptitud física de los escolares de 12 a 18 años del municipio de Montería. Montería: Editorial Paloma.

Silva, I., Vianna, M., Gomes, A. \& Dantas, E. (2008). Diagnóstico do potencial genético físico e somatotipia de uma equipe de futebol profissional Fluminense. Revista Brasileira de Futebol (The Brazilian Journal of Soccer Science), 1(1), 49-58.

Slaughter, M., Lohman, T., Boileau, R., Horswill, C., Stillman, M., Van Loan, M. \& Bemben, D. (1988). Skinfold equations for estimation of body fatness in children and youth. Human Biology, 60(5), 709-723.

Vescovi, J. (2012). Sprint speed characteristics of high-level American female soccer players. Female Athletes in Motion (FAiM) Study. Journal of Science and Medicine in Sport, 15(5), 474-478.

Zalai, D., Bobak, P., Csáki, I., Hamar, P., Myrer, J., Mitchell, U. \& Johnson, A. (2015). Motor Skills, Anthropometrical Characteristics and Functional Movement in Elite Young Soccer Players. Journal of Excercise Sport and Orthopedics, 2(1), 1-7.

Zary, J., Reis, V., Rouboa, A., Silva, A., Fernandes, P. \& Filho, J. (2010). The somatotype and dermatoglyphic profiles of adult, junior and juvenile male Brazilian top-level volleyball players. Science and Sport, 25(3), 146152. 